\title{
FURTHER RESULTS ON GENERALIZED BOTT-DUFFIN INVERSES
}

\author{
Chun Yuan Deng And Hong Ke Du
}

\begin{abstract}
Let $A$ be a bounded linear operator, $P_{\mathscr{M}}$ be an orthogonal projection with range $\mathscr{M}$ and $P_{\mathscr{M}, \mathscr{N}}$ be an idempotent with range $\mathscr{M}$ and kernel $\mathscr{N}$. This paper presents some novel relations between Bott-Duffin inverse $A_{\mathscr{M}}^{+}=P_{\mathscr{M}}\left(A P_{\mathscr{M}}+P_{\mathscr{M}^{\perp}}\right)^{+}$and generalized Bott-Duffin inverse $A_{\mathscr{M}, \mathscr{N}}^{+}=P_{\mathscr{M}, \mathscr{N}}\left(A P_{\mathscr{M}, \mathscr{N}}+P_{\mathscr{N}, \mathscr{M}}\right)^{+}$. Furthermore, the representations for the BottDuffin inverse and generalized Bott-Duffin inverse are presented.
\end{abstract}

Mathematics subject classification (2010): 47A05, 15A09.

Keywords and phrases: Generalized Bott-Duffin inverse, idempotent, orthogonal projection.

\section{REFERENCES}

[1] J. K. BAKSAlary, G. P. H. STYAn, Generalized inverses of partitioned matrices in BanachiewiczSchur form, Linear Algebra Appl. 354 (2002), 41-47.

[2] A. Ben-Israel and T. N. E. Greville, Generalized Inverses: Theory and Applications, WileyInterscience, New York, 1974; 2nd Edition, Springer, New York, 2002.

[3] J. BEN ŞTEZ AND N. ThOME, The generalized Schur complement in group inverses and $(k+1)-$ potent matrices, Linear Multilinear Algebra 54 (2006), 405-413.

[4] R. Bott, R. J. Duffin, On the algebra of network, Trans. Amer. Math. Soc. 74 (1953), 99-109.

[5] S. L. CAmpbell And C. D. Meyer, Generalized Inverses of Linear Transformations, Pitman publishing limited, 1979; SIAM, Philadelphia, 2009.

[6] Y. CHEN, The generalized Bott-Duffin inverse and its applications, Linear Algebra Appl. 134 (1990), 71-91.

[7] G. Chen, G. Liu, Y. Xue, Perturbation analysis of the generalized Bott-Duffin inverse of L-zero matrices, Linear Multilinear Algebra 51 (2003), 11-20.

[8] G. Chen, G. LIU, Y. XUE, Perturbation theory for the generalized Bott-Duffin inverse and its applications, Appl. Math. Comput. 129 (2002), 145-155.

[9] D. S. CvetKović-ILIĆ, J. Chen, AND Z. Xu, Explicit representations of the Drazin inverse of block matrix and modified matrix, Linear Multilinear Algebra 57 (2009), 355-364.

[10] B. Deng, G. Chen, A note on the generalized Bott-Duffin inverse, Appl Math Lett. 20 (2007), 746750.

[11] C. Deng And H. Du, Representations of the Moore-Penrose inverse for a class of 2-by-2 block operator valued partial matrices, Linear Multilinear Algebra 58 (2010), 15-26.

[12] Xiaoji Liu, Chunmei Hu, Yaoming Yu, Further results on iterative methods for computing generalized inverses, Journal of Computational and Applied Mathematics 234 (2010), 684-694.

[13] J. M. Miao, General expressions for the Moore-Penrose inverse of a $2 \times 2$ block matrix, Linear Algebra Appl. 151 (1991), 1-15.

[14] Y. TIAN, Y. TAKANE, More on generalized inverses of partitioned matrices with Banachiewicz-Schur forms, Linear Algebra Appl. 430 (2009), 1641-1655.

[15] Y. Tian, On mixed-type reverse-order laws for the Moore-Penrose inverse of a matrix product, International Journal of Mathematics and Mathematical Sciences 58 (2004), 3103-3116.

[16] G. Wang, Y. Wei And S. QIao, Generalized inverses: Theory and Computions, Science Press, Beijing/New York, 2004. 
[17] Y. WEI, W. XU, Condition number of Bott-Dun inverse and their condition numbers, Appl Math Comput. 142 (2003), 79-97.

[18] Y. TIAN, The equivalence between $(A B)^{+}=B^{+} A^{+}$and other mixed-type reverse-order laws, Int. J. Math. Educ. Sci. Technol. 37 (2006), 331-339.

[19] Q. XU, Moore-Penrose inverses of partitioned adjointable operators on Hilbert $C^{*}-$ modules, Linear Algebra Appl. 430 (2009), 2929-2942.

[20] Q. XU, Y. WEI, AND Y. GU, Sharp norm-estimations for Moore-Penrose inverses of stable perturbations of Hilbert $C^{*}$-module operators, SIAM J. Numer. Anal. 47 (2010), 4735-4758.

[21] Y. XUE, G. CHEN, The expression of the generalized Bott-Duffin inverse and its perturbation theory, Appl Math Comput. 132 (2002), 437-444. 